\title{
Redução da dor crônica associada à escoliose não estrutural, em universitárias submetidas ao método Pilates
}

\author{
Maria Erivânia Alves de Araújo 1, 2, 3 \\ Elirez Bezerra da Silva ${ }^{4}$ \\ Pollyana Coelho Vieira ${ }^{2}$ \\ Samária Ali Cader ${ }^{3,5}$ \\ Danielli Braga de Mello ${ }^{6}$ \\ Estélio Henrique Martin Dantas ${ }^{7}$ \\ ${ }^{1}$ Centro Universitário do Maranhão - UNICEUMA, São Luiz, MA, Brasil \\ ${ }^{2}$ Mestranda do Curso de Pós-Graduação Stricto Sensu, LABIMH da UCB, Rio de janeiro, \\ RJ, Brasil \\ ${ }^{3}$ Laboratório de Biociência da Motricidade Humana, UCB, Rio de Janeiro, RJ, Brasil \\ ${ }^{4}$ Curso de Graduação e Pós-graduação em Fisioterapia e PPGEF da UGF, Rio de \\ Janeiro, RJ, Brasil \\ ${ }^{5}$ Universidade Católica Nossa Senhora de Assunção - Paraguai \\ ${ }^{6}$ Escola de Educação Física do Exército - EsEFEx, Rio de Janeiro, RJ, Brasil \\ 7 Universidade Castelo Branco, Centro de Ciências Biológicas e da Saúde, Programa de \\ Pós Graduação Stricto Sensu em Ciência da Motricidade Humana, Rio de Janeiro, RJ, \\ Brasil
}

\begin{abstract}
Resumo: Objetivo: Avaliar a eficácia do método Pilates na redução da dor crônica associada à escoliose não estrutural. Método: Participaram do estudo ensaio clínico controlado e aleatorizado 31 universitárias, com idade entre 18 e 25 anos, com diagnóstico de escoliose não estrutural e apresentando dor crônica na coluna vertebral. A amostra foi dividida em grupo controle $(n=11)$ que não foi submetido a nenhuma intervenção terapêutica e grupo experimental $(n=20)$ que participou do programa de mecanoterapia pelo método Pilates. A intervenção consistiu de vinte e quatro sessões. Os movimentos foram orientados de acordo com a convexidade da escoliose de cada indivíduo. Foi utilizado o teste de Adams, radiografia panorâmica da coluna vertebral (pré tratamento) e um questionário mediante a "Escala de dor percebida CR 10 de Borg" (pré e pós tratamento). O tratamento estatístico utilizado foi a ANOVA 2x2 de medidas repetidas, seguida do teste post hoc de Tukey. Resultados: Os resultados identificaram diminuição significativa de $66 \%$ da dor no grupo experimental $(P=0,0002)$. Conclusão: Pode-se verificar que 0 programa de exercícios utilizando-se o método Pilates para jovens com escoliose não estrutural, apresentando dor na coluna vertebral é eficaz, pois houve redução da intensidade de dor.
\end{abstract}

Palavras-chave: Cinesioterapia, Mecanoterapia, Postura.

\section{Reduction of the chronic pain associated to the scoliosis non structural, in university students submitted to the Pilates method}

\begin{abstract}
Objective: To evaluate the effectiveness of the Pilates method in the reduction of the chronic pain associated to a non structural scoliosis. Method: 31 academical subjects participated in the clinical aleatorical controlled experiment study, under age between 18 and 25 years, with diagnosis of non structural scoliosis and presenting chronic pain in the spine. The sample was divided into: control group $(n=11)$ that the was not submitted to any therapeutic intervention and experimental group $(n=20)$ that participated of the mecanotherapy program for the Pilates method. The intervention consisted of twenty-four sessions. The movements were guided in agreement with the convexity of each individual's scoliosis. The test of Adams, panoramic x-ray of the spine was used (pre treatment) and a questionnaire by the "Scale of noticed pain CR 10 of Borg" (pre and post treatment). The used statistical treatment was ANOVA 2x2 of repeated measures with the test post hoc of Tukey. Results: Findings demonstrated a significant decrease of $66 \%$ of the pain for the experimental group $(P=0,0002)$. Conclusion: It can be observed that the exercise program using the Pilates method to young people is effective and improved the reduction of chronic pain among young subjects presenting non structural scoliosis.
\end{abstract}

Key Words: Kinesiotherapy. Mecanotherapy. Posture. 


\section{Introdução}

Em alguma época da vida, de 70 a $85 \%$ de todas as pessoas sofrerão de dores nas costas. As dores crônicas da coluna vertebral devem ser tratadas como um problema de saúde pública (SILVA; FASSA; VALLE, 2004), pois esta morbidade é uma das mais importantes causas de absenteísmo nos países desenvolvidos (GOUVEIA; GOUVEIA, 2008).

A dor possui um papel de alerta para comunicar ao organismo que algo está errado e, em se tratando de uma dor crônica, ela gera no organismo um estresse e até mesmo, uma incapacidade física. Portanto, a dor é um problema que demanda cuidados imediatos, e a melhor estratégia para se eliminar qualquer tipo de dor é a remoção do fator causal (HEREDIA; RODRIGUES, 2008).

Uma das causas de dores na coluna vertebral são os problemas posturais que podem gerar desde desconfortos relativamente leves até lesões mais graves, como as doenças osteoarticulares (PACCINI; CYRINO; GLANER, 2007). A escoliose é uma das deformidades da coluna que se apresenta como um desvio lateral (EVANS, 2003).

De acordo com sua etiologia, pode ser classificada em estrutural e não estrutural. A escoliose estrutural pode ser idiopática (sem causa aparente), neuromuscular e osteopática. Já a não-estrutural pode ser causada pela assimetria de membros inferiores, espasmo ou dor muscular da coluna vertebral por compressão de raiz nervosa ou outra lesão na coluna e, ainda, pelos maus hábitos posturais (BONORINO; BORIN; SILVA, 2007).

Um dos mecanismos causais são devidos ao tensionamento dos músculos multifidos contralaterais, na tentativa de manter 0 alinhamento ao carregar uma mochila, por exemplo, causando assim, fadiga e dores musculares. Adicionado a isso, poderá ocorrer o comprometimento de outros músculos relacionados ao alinhamento látero-lateral da coluna, como os oblíquos abdominais, tornando a escoliose permanente na postura do indivíduo (SACCO et al., 2003). Estes músculos se contraem juntamente com o quadrado lombar em uma ação unilateral, produzindo uma inflexão do tronco do lado da sua contração (KAPANDJI, 2000).
Segundo Rydeard, Leger e Smith (2006), a dor crônica da coluna vertebral pode ocorrer também pela alteração do controle neuromuscular de estabilidade do tronco e a eficiência do movimento; e esta estabilidade é alcançada pelo controle da região pélvico-lombar pela ativação específica dos músculos extensores do quadril, os glúteos máximos.

Nas escolioses álgicas do adulto, o alongamento muscular pode gerar um importante efeito analgésico. Teoriza-se que os problemas relacionados com a escoliose podem ocorrer como resultado do desequilíbrio do corpo e padrões de movimentos "preferidos". Uma área fraca ou desalinhada pode resultar numa propensão à compensação ou desenvolvimento de outra área (Blum, 2002).

Almeida e Jabur (2006) relatam que é necessário exercitar-se quando se sofre de dor crônica, pois o exercício pode diminuir problemas como músculos encurtados, perda de mobilidade articular e fraqueza, que contribuem para a dor; e prevenir as complicações musculoesqueléticas secundárias da dor, como a fraqueza (astenia). 0 tratamento da dor crônica é dirigido à fonte de dor e a quaisquer deficiências ou limitações funcionais musculoesqueléticas, bem como a quaisquer problemas que podem ser prevenidos, identificados durante o processo de avaliação. $O$ alongamento, portanto, tem sido usado para diminuir a dor e a tensão muscular.

O método Pilates consiste na realização de exercícios físicos, que utiliza a gravidade e recursos mecanoterapêuticos como as molas, que atuam como resistência durante a execução do movimento, como também no auxílio do próprio movimento (RODRIGUES et al., 2010).

Esse método caracteriza-se por movimentos projetados de forma que os executantes mantenham a posição neutra da coluna vertebral, minimizando o recrutamento muscular desnecessário, prevenindo a fadiga precoce e a diminuição da estabilidade corporal. $\mathrm{O}$ treinamento com esse método visa melhorar a flexibilidade geral do corpo, a força muscular, a postura e a coordenação da respiração com o movimento, sendo, portanto, esses fatores essenciais no processo de reabilitação postural (SEGAL; HEIN; BASFORD, 2004).

Os exercícios envolvem contrações concêntricas e excêntricas e, principalmente, 
isométricas, com ênfase no que Joseph Humbertus Pilates, o criador do método Pilates, denominou power house (ou centro de força). Esse centro de força é composto pelos músculos abdominais, glúteos e paravertebrais lombares, que têm a função de estabilizar estática e dinamicamente o corpo (SILVA; MANNRICH, 2009).

A literatura aponta que o método Pilates tem alguns benefícios que ajudam a prevenir lesões e proporcionar um alívio de dores crônicas. Dentre esses benefícios podemos citar: estimular a circulação, melhorar o condicionamento físico, a flexibilidade, a amplitude muscular, o alinhamento postural, melhorar os níveis de consciência corporal e a coordenação motora (SACCO et al., 2005).

Portanto, o objetivo deste estudo foi verificar a eficácia dos exercícios mecanoterapêuticos utilizando o método de Pilates sobre a intensidade da dor crônica em universitárias com escoliose não estrutural.

\section{Materiais e método}

O desenho adotado foi de estudo ensaio clínico controlado e aleatorizado (THOMAS; NELSON; SILVERMAN, 2007).

\section{Sujeitos}

Participaram do estudo 31 universitárias do Centro Universitário do Maranhão (UNICEUMA), voluntárias, com $22 \pm 2$ aos de idade, todas com diagnóstico de escoliose não estrutural (dorsolombar destra convexa ou dorsolombar sinistra convexa), com ângulo de Cobb $7 \pm 3$ graus e referindo dor crônica na coluna vertebral (torácica e/ou lombar) há $36 \pm 16$ semanas.

O diagnóstico de escoliose dorsolombar convexa não estrutural foi dado por fisioterapeuta após exame direto da coluna vertebral, execução do teste de Adams e exames de raios- $X$, seguido do cálculo do ângulo de Cobb.

Foram excluídas do estudo aquelas que apresentaram gibosidade por ocasião do teste de Adams ou que faltaram a alguma sessão de Pilates.

As 31 universitárias foram alocadas aleatoriamente utilizando uma série de números aleatórios gerados por sorteios, sendo que as participantes que ficassem com os números ímpares foram para o $\mathrm{GE}(\mathrm{n}=20$, com idades de $21 \pm 01$ anos, dor crônica de $5 \pm 2$ de intensidade e ângulos de Cobb de $8 \pm 3$ graus) que participou efetivamente do programa de mecanoterapia pelo método de Pilates, e as que ficassem com os números pares, foram para o $\mathrm{GC}(\mathrm{n}=11$, com idades de $22 \pm 2$ anos, dor de $4 \pm 2$ de intensidade e ângulos de Cobb de $7 \pm 3$ graus), que não foi submetido a nenhuma intervenção terapêutica. Os grupos GE e GC apresentaram homogeneidade em relação a idade, a dor crônica e ângulo de Cobb $(P>0,05)$.

$\mathrm{O}$ presente estudo atendeu às normas para a realização de investigações com seres humanos, conforme todos os procedimentos da Declaração de Helsinki (WORLD MEDICAL ASSOCIATION, 2009), e foi aprovado pelo Comitê de Ética em Pesquisa da Universidade Castelo Branco/RJ sob o no 0143/2008, em 10 de Novembro de 2008.

\section{Avaliação da escoliose dorsolombar convexa}

Inicialmente, a fisioterapeuta realizou a inspeção (observação direta) da coluna vertebral das participantes para detectar a presença de escoliose dorsolombar destra convexa (EDLDC) ou escoliose dorsolombar sinistra convexa (EDLSC). Em seguida, foi realizado o teste de Adams para observar se a escoliose era não estrutural. Neste procedimento, a participante ficou em pé, descalço e sem camisa, com os membros inferiores estendidos realizando uma flexão anterior do tronco para se identificar a presença ou não de gibosidade (GOLDBERG et al., 2008).

Posteriormente, foi examinada a imagem radiográfica panorâmica da coluna vertebral das participantes. Este exame foi realizado na clínica SOS TRAUMA em São Luís, por um técnico de raios- $X$, que utilizou um instrumento da marca Emic, modelo EMERALD 130-3 (USA) com o objetivo de confirmação de uma real existência de uma EDLDC ou EDLSC. No exame da imagem radiográfica, a fisioterapeuta além de confirmar a escoliose observada na inspeção anterior mediu o ângulo de Cobb, que foi obtido pelo traço de uma linha paralela à borda superior da vértebra limítrofe superior e outra na borda inferior da vértebra limítrofe inferior da escoliose. A intersecção destas duas linhas determinou a angulação do desvio da coluna vertebral (MODI et al., 2008). Este ângulo foi medido com um goniômetro da marca CARCI (Brasil). 


\section{Avaliação da dor crônica na coluna vertebral}

A classificação da dor como crônica foi feita na anamnese a partir das perguntas: "Você sente dor na coluna vertebral? Em qual região? Há quanto tempo você sente esta dor?". A intensidade de dor crônica na coluna vertebral foi medida pela Escala percebida CR10 de Borg, que varia de 0 a 10 (BORG, 1982). Foi explicado para as participantes que 0 corresponde a ausência de dor e 10, a dor extremamente forte, e pedido que ela apontasse 0 número inteiro entre 0 e 10 que mais se associava com a intensidade de dor sentida. Os dois grupos GE e GC tiveram a dor medida antes e após 12 semanas de tratamento com Pilates.

\section{Método Pilates}

O grupo experimental realizou 24 sessões de tratamento pelo programa de exercícios mecanoterapêuticos, utilizando-se a metodologia de Pilates, com atendimentos de duas vezes por semana durante 60 minutos por sessão, no Studio de Reeducação Postural em São Luís.

O protocolo de exercícios foi dividido em três etapas: preparação (aquecimento, seguido de exercícios de alongamento), parte específica e volta à calma.

Preparação: o aquecimento consistiu de uma caminhada de 8 minutos e de intensidade conforme situação mais confortável para cada participante em uma esteira elétrica da marca YOZDA® (Brasil) ou elíptico da marca MOVIMENT® (Brasil), seguido de movimentos de alongamento no solo em um tempo de 5 minutos (5 repetições para cada movimento). As participantes foram orientadas a realizar os seguintes alongamentos:

- Alongamento da coluna para frente: paciente sentada com a coluna ereta, pernas estendidas e pés em flexão plantar. A mesma foi orientada a levar os membros superiores para frente, em um movimento de flexão anterior do tronco. Os objetivos deste movimento são alongar a cadeia muscular posterior e mobilizar a coluna vertebral.

- Rolamento por cima: paciente deitada em decúbito dorsal com os membros superiores ao lado do corpo. Ela foi orientada a elevar as duas pernas estendidas sobre a cabeça até as pontas dos pés tocarem no solo, e em seguida, desenrolar a coluna lentamente, descendo vértebra por vértebra, sem deixar as pernas encostarem-se ao solo, mantendo-as à $90^{\circ}$ com o corpo. Os objetivos deste movimento são alongar a cadeia posterior, mobilizar a coluna vertebral e fortalecer o reto do abdômen, oblíquo externo, reto femoral e tensor da fáscia lata.
- "Prece Maometana": a partir da posição de quatro apoios, a paciente foi orientada a alongar a coluna, empurrando suas mãos contra o solo com os membros superiores estendidos, e abaixando a coluna para que os músculos isquiostibiais ficassem próximos aos glúteos. Os objetivos deste movimento são alongar paravertebrais torácicos, lombares e glúteos e mobilizar a coluna vertebral.

- Puxar a perna de frente: em posição de quatro apoios, a paciente que apresentava EDLSC foi orientada a elevar o membro superior direito e o membro inferior direito, mantendo o tronco alinhado e evitando a rotação de quadril, e quem apresentava EDLDC foi orientada a elevar o membro superior esquerdo e o membro inferior esquerdo. O objetivo deste movimento é alongar a concavidade da coluna vertebral.

Parte específica: foram utilizados exercícios com bolas Suíças da marca FlexBall Quark® (Brasil) e equipamentos próprios da técnica de Pilates da marca $D \& D$ Pilates $\AA$ (Brasil). Nas duas primeiras semanas (4 atendimentos), houve um período de adaptação dos indivíduos com os movimentos associados à respiração, a fim de buscar a execução correta dos exercícios orientados. Esta familiarização com os exercícios fizeram parte das 24 sessões de Pilates.

Nesta etapa, foram realizados doze exercícios (10 repetições cada exercício) descritos a seguir:

\section{1- Rolamentos de quadril com bola grande $(65 \mathrm{~cm}$} de diâmetro)

Posição inicial: Em decúbito dorsal, com as pernas apoiadas em cima da bola e os membros superiores ao lado do corpo com os ombros relaxados.

Sequência do movimento: 1. Inspirar na posição inicial; 2. Expirar, direcionando o umbigo em direção à coluna, e gradativamente, levantar a pélvis do solo até alinhar o corpo; 3 . Inspirar enquanto estiver com a pélvis elevada; 4 . Expirar, enquanto for relaxando 0 tórax, descendo vértebra por vértebra.

Objetivos: Fortalecer os glúteos e desenvolver um equilíbrio.

\section{2- Abdominal Invertido com bola $(55 \mathrm{~cm}$ de diâmetro)}

Posição Inicial: Em decúbito dorsal, flexionar os joelhos, apertando a bola com a parte posterior das pernas, coxas e calcanhares. Membros superiores ao lado do corpo.

Sequência do movimento: 1. Inspirar na posição inicial; 2. Expirar, trazendo os joelhos até o tórax, suspendendo a bola do solo; 3 . Inspirar, permanecendo na posição anterior; 4. Expirar, voltando a bola ao solo.

Objetivos: Fortalecer a região infra-abdominal e os músculos isquiostibiais. 


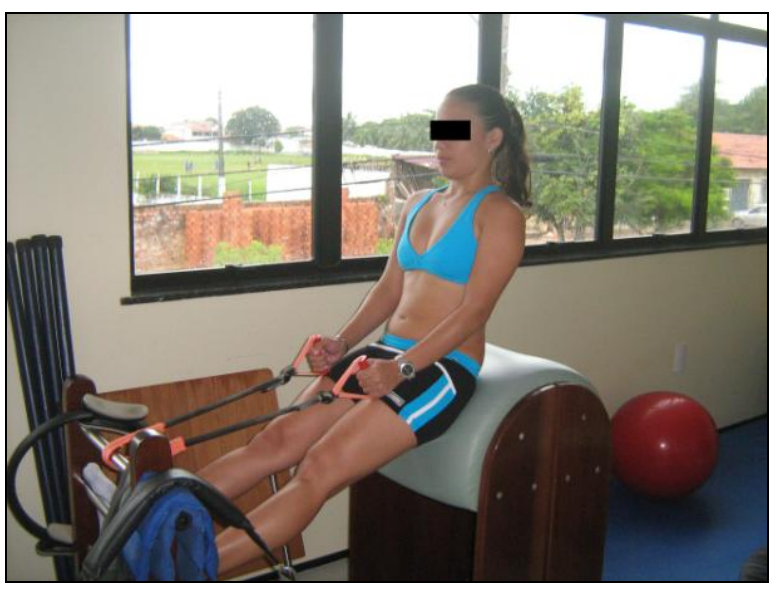

Figura 1. Movimento realizado no Ladder-Barrel para fortalecimento do músculo reto-abdominal

\section{3- Subir até sentar (Figura 1)}

Posição Inicial: Deitada em decúbito dorsal sobre - Ladder-Barrel, cotovelos estendidos, mãos segurando acessório de apoio e pés apoiados no espaldar.

Sequência do Movimento: 1. Inspirar na posição inicial; 2. Expirar, levando o queixo em direção ao peito e retirando as escápulas do Barrel até sentar; 3. Inspirar; 4. Expirar, retornando à posição inicial, enrolando a coluna.

Objetivos: Fortalecer o reto do abdômen.

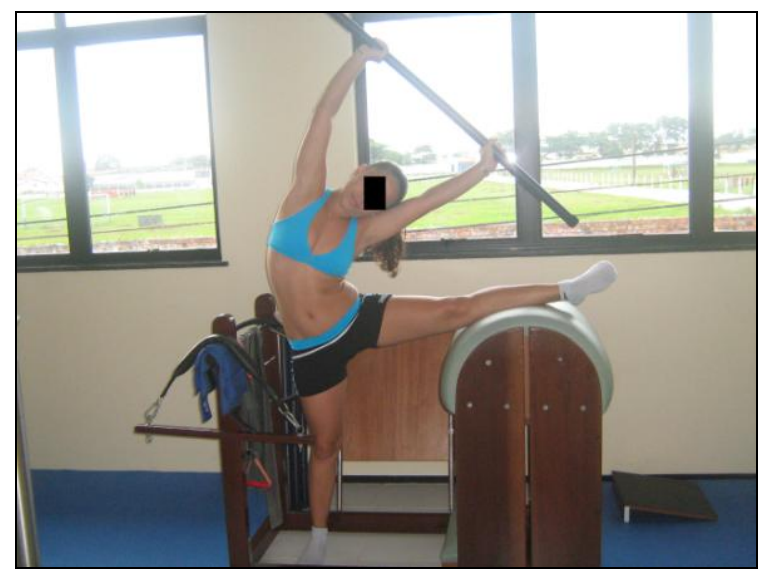

Figura 2. Movimento realizado no Ladder-Barrel para correção de uma escoliose dorsolombar sinistra convexa

\section{4- Movimento lateral do tronco (Figura 2)}

Posição Inicial: Em pé, lateralmente ao LadderBarrel, com um membro inferior apoiado sobre o mesmo em abdução e rotação externa; mãos segurando um bastão acima do corpo.

Sequência do Movimento: 1. Inspirar na posição inicial; 2. Expirar, realizando uma flexão lateral do tronco de acordo com a convexidade de cada escoliose; 3. Inspirar; 4. Expirar, retornando à posição inicial.

Objetivos: Alongar cadeia muscular lateral de acordo com a convexidade da escoliose.

5- Sereia no Reformer com 2 molas de 0,0240 Kgf cada posicionadas nas argolas cujo braço de força ficou regulado na resistência máxima

Posição Inicial: Paciente sentada de lado com as pernas cruzadas sobre o Reformer com uma mão na barra móvel.

Sequência do movimento: 1. Inspirar na posição inicial; 2. Expirar, empurrando a barra e simultaneamente, levando o braço oposto em direção à cabeça fazendo um "C" lateral com a coluna; 3. Inspirar; 4. Expirar, retornando à posição inicial.

Objetivos: Alongar cadeia muscular lateral de acordo com a convexidade de cada escoliose.

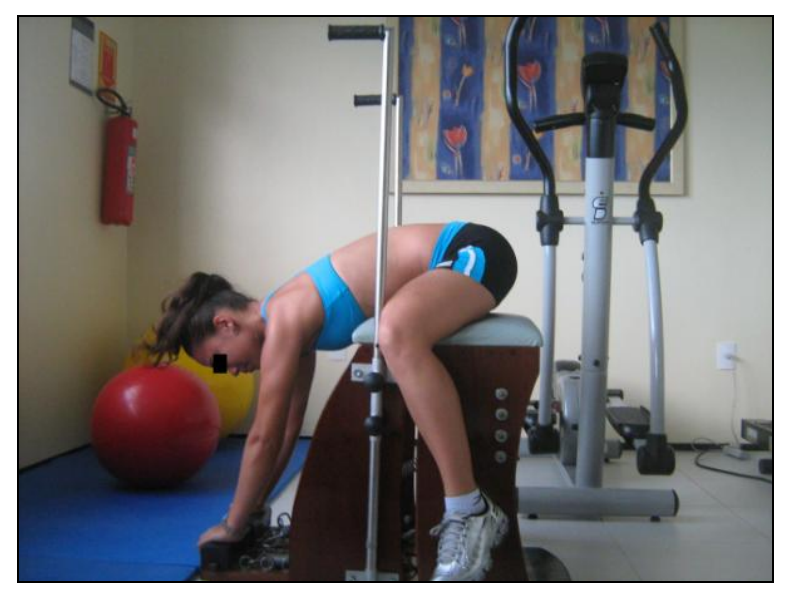

Figura 3. Movimento realizado no Step-Chair em extensibilidade sub-máxima dos músculos adutores do quadril e paravertebrais

6- Cavalo (Figura 3) no Step-Chair com 1 mola de 0,1410 Kgf posicionada nas argolas de ajustes de maior resistência

Posição Inicial: Sentada na parte de trás do acento do Step-Chair, quadris abduzidos e mãos no step.

Sequência do Movimento: 1. Inspirar na posição inicial; 2. Expirar, empurrando o step para baixo; 3. Inspirar; 4. Expirar, retornando à posição inicial desenrolando a coluna.

Objetivos: Mobilizar a coluna e alongar paravertebrais tóraco-lombares e adutores do quadril.

7- Série de perna supino no Cadillac com 2 molas de 0,0150 Kgf cada situadas nas argolas superiores e laterais do aparelho

Posição Inicial: Participante em decúbito dorsal no aparelho Cadillac, com as alças dos pés, membros inferiores em flexão de quadril à $90^{\circ} \mathrm{e}$ membros superiores ao lado do corpo.

Sequência do movimento: 1. Inspirar na posição inicial; 2. Expirar, realizando extensão de quadris, mantendo os tornozelos juntos e joelhos em extensão; 3. Inspirar; 4. Expirar, retornando à posição inicial.

Objetivos: Fortalecer glúteo máximo, isquiostibiais e adutores do quadril.

8- Extensão de perna no Cadillac com 2 molas de 0,0150 Kgf cada situadas nas argolas superiores e laterais do aparelho

Posição Inicial: Participante em decúbito dorsal no aparelho Cadillac, com as alças dos pés, joelhos 
e quadris flexionados à $90^{\circ}$ e membros superiores ao lado do corpo.

Sequência do Movimento: 1. Inspirar na posição inicial; 2. Expirar, realizando extensão de joelhos e quadris, mantendo os tornozelos juntos; 3 . Inspirar; 4. Expirar, retornando à posição inicial. Objetivos: Fortalecer glúteo máximo e quadríceps.

9- Braços para cima e para baixo no Reformer com 2 molas de 0,0240 Kgf cada posicionadas nas argolas cujo braço de força do aparelho ficou regulado na resistência máxima

Posição Inicial: Participante em decúbito dorsal no aparelho Reformer, segurando as alças de mãos, com os ombros fletidos à $90^{\circ}$ e mantendo joelhos e quadris em flexão de $90^{\circ}$.

Sequência do movimento: 1. Inspirar na posição inicial; 2. Expirar, realizando extensão de ombros; 3. Inspirar; 4. Expirar, retornando à posição inicial. Objetivos: Fortalecer grande dorsal, redondo maior, peitoral maior e deltóide posterior.

10-Braços (Tríceps) no Reformer com 2 molas de 0,0240 Kgf cada posicionadas nas argolas cujo braço de força do aparelho ficou regulado na resistência máxima

Posição Inicial: Participante em decúbito dorsal no aparelho Reformer, segurando as alças de mãos, com os cotovelos fletidos à $90^{\circ}$ e mantendo joelhos e quadris em flexão de $90^{\circ}$.

Sequência do movimento: 1. Inspirar na posição inicial; 2. Expirar, realizando extensão de cotovelos; 3. Inspirar; 4. Expirar, retornando à posição inicial.

Objetivos: Fortalecer tríceps braquial e ancôneo.

11- Rolando para trás no Cadillac com 2 molas de 0,0150 Kgf cada posicionadas nas argolas superiores do aparelho

Posição Inicial: Paciente sentada no aparelho Cadillac, com os pés apoiados nas hastes laterais, segurando a barra móvel.

Sequência do movimento: 1. Inspirar, na posição inicial; 2. Expirar, realizando uma flexão anterior da cabeça sobre o peito, e ir rolando o tronco lentamente para trás, mobilizando vértebra por vértebra, até deitar completamente; 3. Inspirar; 4. Expirar, realizando novamente flexão anterior da cabeça, e lentamente desenrolar a coluna para cima, até sentar.

Objetivos: Melhorar a mobilidade da coluna vertebral e o controle abdominal.

12- Alongamento da coluna no Cadillac com 2 molas de 0,0150 Kgf cada posicionadas nas argolas superiores do aparelho

Posição Inicial: Paciente em pé sobre o Cadillac com as mãos na barra fíxa.

Sequência do movimento: 1. Inspirar na posição inicial; 2. Expirar, enrolando a cervical e o tronco para frente, realizando uma flexão anterior do tronco até a amplitude máxima, retificando a coluna; 3. Inspirar; 4. Expirar, retornando à posição inicial.

Objetivos: Alongar a cadeia posterior.
Os movimentos aconteceram em uma velocidade lenta e constante, trabalhando as articulações em uma amplitude máxima de flexibilidade, sendo estimulada a precisão dos movimentos, a propriocepção e a concentração durante a execução. Todos os exercícios foram realizados pedindo-se que os indivíduos mantivessem ainda, a pelve em posição neutra, abdômen sempre contraído e cabeça centralizada, mantendo a lordose fisiológica da cervical. Alguns movimentos foram orientados de acordo com a convexidade da escoliose de cada participante.

Volta à calma: este procedimento foi composto por 3 movimentos, teve a duração de 5 minutos e foram realizados 3 repetições para cada movimento. Estes foram executados suavemente, buscando um estado de relaxamento, visando à recuperação metabólica e a descontração da musculatura estimulada (Relaxamento nas bolas de 65 e $75 \mathrm{~cm}$ de diâmetro):

1. Participante deitada de lado na bola de acordo com a convexidade da escoliose;

2. Em seguida, deitada em prono na bola, mantendo o apoio das pontas dos pés e das mãos;

3. E por último, deitada em supino na bola, mantendo os pés apoiados e os membros superiores para trás.

Todo o procedimento de aplicação dos exercícios foi realizado pela mesma fisioterapeuta do Studio de Reeducação Postural, que possui certificado de qualificação como instrutora do método Pilates no Brasil.

Semanalmente, o GC tinha um encontro com a pesquisadora que se assegurava que o mesmo se mantinha sem nenhuma intervenção terapêutica, mesmo apresentando dor crônica na coluna vertebral.

Após as 24 sessões da aplicação do método (três meses), foram realizadas as reavaliações da intensidade de dor crônica da coluna vertebral, respeitando-se os mesmos procedimentos adotados durante a avaliação inicial.

\section{Análise dos dados}

Os resultados são apresentados em valores de média \pm desvio-padrão. A hipótese de diferença de dor intra e inter grupos GE e GC foi testada pela estatística inferencial ANOVA 2x2, com medidas repetidas, sendo o primeiro fator os grupos GE e GC e o segundo fator as medidas pré e pós, seguida do teste post hoc de Tukey para amostras diferentes. O nível de significância adotado foi de $5 \%(P<0,05)$. Todos os testes estatísticos utilizados pertenciam ao programa Statistica 6.0, StatSoft, Inc, 1984-2001. A análise 
foi realizada por outra pessoa diferente daquela que aplicou o tratamento e mediu a dor.

\section{Resultados}

As médias e desvios-padrões da intensidade de dor dos grupos controle pré e pós tratamento foram de $4,4 \pm 2,3$ e $3,8 \pm 2,7$, respectivamente e do grupo Pilates pré e pós tratamento foram de $5,3 \pm 1,5$ e $1,8 \pm 1,7$, respectivamente.

A ANOVA 2x2 de medidas repetidas das dores apresentaram diferenças significativas na interação dos grupos com o momento da medida $\left(\mathrm{F}\left({ }_{1}, 29\right)=13,693, P=0,0009\right)$. O teste post hoc de Tukey detectou diminuição significativa da dor no grupo Pilates $(P=0,0002)$. O mesmo não ocorreu no grupo controle $(P=0,84)$. Os grupos não apresentaram diferenças nas dores antes e após o tratamento $(P>0,80)$ (Figura 4).

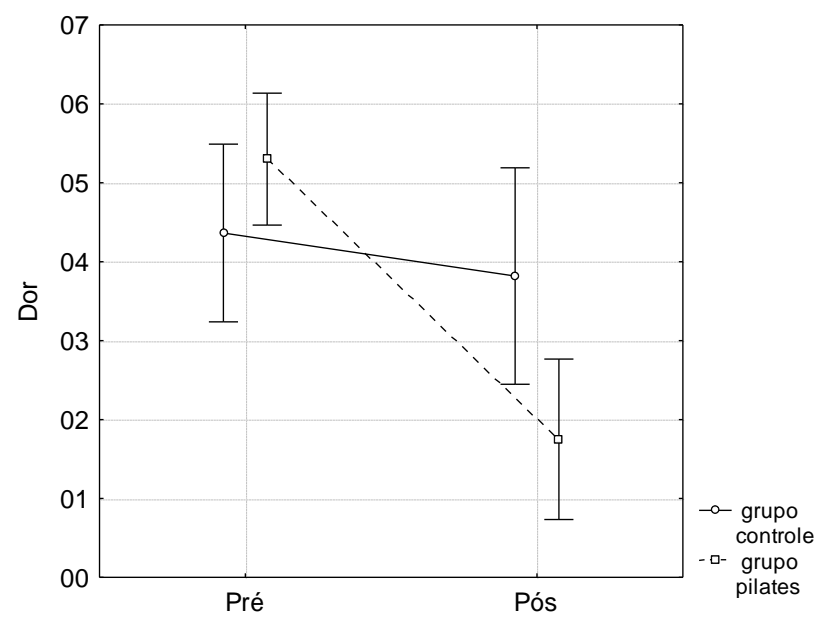

Figura 4. Dores dos grupos controle e Pilates antes e depois do tratamento com Pilates $\left(F_{(1,29)}\right.$ $=13,693, P=0,0009)$. Dor Pós-Pilates significativamente menor que a dor Pré-Pilates $(P$ $=0,0002$ )

\section{Discussão}

Uma das ferramentas utilizadas para minimizar a dor na coluna vertebral, são os diversos exercícios posturais utilizados pela fisioterapia. $O$ método Pilates, de interesse neste estudo, mostrou-se eficaz como uma das formas de tratamento fisioterapêutico em jovens que referiam dor na coluna vertebral em decorrência da escoliose não estrutural. Conforme se pode observar na Figura 4, os resultados mostraram a redução significativa em $66 \%$ na intensidade de dor, provavelmente em função dos efeitos do programa de exercícios mecanoterapêuticos pelo método Pilates.

Diante destes resultados, é possível
argumentar como os exercícios
mecanoterapêuticos pelo método Pilates podem
ter diminuído a intensidade de dor do grupo

submetido a 24 sessões. É provável que os exercícios agiram sobre o controle postural e estimularam 0 trabalho dos músculos estabilizadores da coluna. Esta afirmação pode ser reforçada pelo estudo de Endleman e Critchley (2008), que utilizaram ultrasom para mostrar que 18 mulheres e 8 homens praticantes de Pilates por mais de 6 meses apresentaram maior espessura dos músculos transverso e oblíquos internos abdominais. Estes músculos, juntamente com os multifidos, são músculos estabilizadores da coluna vertebral. Outro raciocínio importante que podemos relacionar com a diminuição da intensidade da dor na coluna vertebral é o fato de que a maioria dos exercícios executados pela metodologia de Pilates é realizada na posição deitada, havendo diminuição dos impactos nas articulações de sustentação do corpo na posição ortostática e, principalmente, na coluna vertebral, permitindo a recuperação das estruturas musculares, articulares e ligamentares, particularmente da região sacrolombar (Kolyniak, Cavalcanti e Aoki, 2004).

Richardson e Jull (1995), ao aplicarem exercícios de co-contração para a reeducação do transverso do abdome em pacientes com dor lombar, seguiram os mesmos princípios do método Pilates e observaram que os exercícios realizados com contração isométrica dos estabilizadores da coluna lombar são mais eficazes na melhora da dor. O'Sullivan et al., (1997), comparou pacientes com lombalgia que utilizaram exercícios específicos também de cocontração dos músculos abdominais profundos e multifido lombar, baseados no trabalho de Richardson, com outros que realizaram tratamentos diversos com exercícios tradicionais. Aqueles que fizeram os exercícios de reeducação do transverso apresentaram resultados estatisticamente significativos na intensidade da dor, apontando efetividade na reeducação do transverso abdominal. Novamente, O'Sullivan, em 2000, utilizou os mesmos princípios de treinamento, comparando padrões de instabilidade lombar e obteve bons resultados melhorando a dor crônica e também havendo a correção postural.

Nossos achados corroboram com Maher (2004), o qual realizou um estudo sobre tratamento da dor lombar e concluiu que os exercícios de contração dos músculos multifidos e transverso abdominal associada à respiração são clinicamente importantes para o ganho de movimento e diminuição da dor. Gladwell et al. (2006) revelou que o método Pilates se apresentou como exercício específico de estabilização dos músculos abdominais e que 
incorporou atividades funcionais que melhoraram a dor crônica nas costas de causa não específica em indivíduos ativos quando comparados aos que não passaram por nenhuma intervenção.

Outro estudo que mostrou resultado eficaz quanto à redução da dor crônica da coluna vertebral foi realizado por Donzelli et al. (2006). Eles utilizaram duas técnicas de tratamento com tratamentos randomicamente controlados: a metodologia de Pilates e a Escola de Postura. No grupo de Pilates, os valores obtidos (80,95\%) com a Escala Oswestry mostrou uma queda acentuada na intensidade da dor no primeiro mês comparado ao grupo da Escola de Postura $(63,64 \%)$. O resultado mais eficaz foi obtido com o grupo Pilates, porque os pacientes aderiram melhor a esse programa de tratamento. Esse fato pode ser explicado pela simplicidade e boa adaptabilidade que o Pilates tem a cada tipo de paciente, por se tratar de um serviço personalizado mesmo quando a sessão é feita em grupo, além da originalidade e da variedade de exercícios que pode encorajar a pro-atividade e atitude positiva em relação ao tratamento. Para que isto aconteça, é necessário que os pacientes percebam os benefícios adquiridos pelos exercícios durante o tratamento. Essa percepção dos benefícios ficou registrada na pesquisa realizada por Gagnon et al. (2009), onde um dos objetivos mais importantes de um programa de formação multidisciplinar para dor lombar crônica foi fazer com que os pacientes se tornassem conscientes dos benefícios da prática regular de uma atividade física.

Segundo Sekendiz et al. (2007), os exercícios do método Pilates tornaram-se mais conhecidos popularmente nas duas últimas décadas. No entanto, houve poucas pesquisas sobre os benefícios do método em pessoas sedentárias, uma vez que havia muitas informações desses benefícios em bailarinos. Os resultados do presente estudo contribuirão para aumentar a quantidade de estudos de Pilates com pessoas sedentárias, já que as participantes deste estudo eram jovens universitárias sedentárias com dor crônica na coluna vertebral.

\section{Conclusão}

Foi possível verificar que um programa de exercícios mecanoterapêuticos, utilizando a metodologia de Pilates, foi eficaz na diminuição da dor em jovens universitárias com escoliose não estrutural.

Foi observada uma escassez de experimentos controlados e randomizados relacionando os efeitos dos exercícios mecanoterapêuticos pelo método Pilates sobre as alterações posturais e a dor na coluna vertebral.

A utilização deste protocolo de tratamento para diminuir a intensidade da dor crônica, pode ser relevante no processo de reabilitação das algias da coluna vertebral. Porém, recomendam-se futuros estudos para investigar o efeito do método Pilates na diminuição das escolioses posturais.

\section{Referências}

ALMEIDA, T. T.; JABUR, M. N. Mitos e verdades sobre flexibilidade: reflexões sobre o treinamento de flexibilidade na saúde dos seres humanos.

Revista motricidade, n. 3, p. 337-344, 2006.

BLUM, C. L. Chiropractic and pilates therapy for the treatment of adult scoliosis. Journal of Manipulative and Physiological Therapeutics, v. 25, n. $4,2002$.

BONORINO, K. C.; BORIN, G. S.; SILVA, A. H. Tratamento para escoliose através do método isostretching e uso de bola suíça. Revista Cinergis, v. 8, n. 2, p. 1-6, 2007.

BORG, G. Psychophysical bases of perceived exertion. Medicine and Science in Sports and Exercise, n. 14, p. 377-381, 1982.

DONZELLI, S. et al. Tho differents technicques in the rehabilitation treatment of low back pain: a randomized controlled trial. Europa medicophysica, n. 42, p. 205-210, 2006.

ENDLEMAN, I.; CRITCHLEY, D. J. Transversus Abdominis and Obliquus Internus Activity During Pilates Exercises: Measurement With Ultrasound Scanning. Archives of physical medicine and rehabilitation, n. 89, p. 2205-2212, 2008.

\section{EVANS, R. C. Exame físico ortopédico} ilustrado. 2. ed. São Paulo: Manole, 2003.

FERREIRA, D. M. A. et al. Rastreamento escolar da escoliose: medida para o diagnóstico precoce. Revista brasileira de crescimento e desenvolvimento humano, v. 19, n. 3, p. 357368, 2009.

GAGNON, S. et al. Multicenter multidisciplinary training program for chronic low back pain: French experience of the Renodos back pain network (Re'seau Nord-Pas-de-Calais du DOS). Annals of Physical and Rehabilitation Medicine, n. 52, p. 3-16, 2009.

GLADWELL, V. et al. Does a Program of Pilates Improve Chronic Non-Specific Low Back Pain? Journal of sport rehabilitation, n. 15, p. 338350, 2006.

GOLDBERG, C. J. et al. Scoliosis: a review. Pediatric surgery international, n. 24, p. 129144, 2008. 
GOUVEIA, K. M. C.; GOUVEIA, E. C. O músculo transverso abdominal e sua função de estabilização da coluna lombar. Fisioterapia em Movimento, v. 21, n. 23, p. 45-50, jul./set. 2008.

HEREDIA, E. P.; RODRIGUES, F. O Tratamento de Pacientes com Fibrose Epidural pela Reeducação Postural Global - RPG. Revista Brasileira de Neurologia, v. 44, n. 3, p. 19-26, 2008.

KAPANDJI, A. I. Fisiologia Articular: esquemas comentados de mecânica humana. Tronco e Coluna Vertebral. vol. III. 5. ed. São Paulo: Panamericana, 2000.

KOLYNIAK, I. E. G. G.; CAVALCANTI, S. M. B.; AOKI, M. S. Avaliação isocinética da musculatura envolvida na flexão e extensão do tronco: efeito do método Pilates ${ }^{\circledR}$. Revista Brasileira de Medicina do Esporte, n. 10, p. 487-490, 2004.

MAHER, C. G. Effective physical treatment for chronic low back pain. Orthopedic Clinics of North America, v. 35, n. 1, p. 57-64, 2004.

MODI, H. N.; CHEN, T.; SUH, S. W.; MEHTA, S.; SRINIVASALU, S.; YANG, J. H.; SONG, H. R. Observer reliability between juvenile and adolescent idiopathic scoliosis in measurement of stable Cobb's angle. European Spine Journal, n. 18, p. 52-58, 2008.

O'SULLIVAN, P. B. et al. Evaluation of specific stabilizaing exercise in the treatment of chronic low back pain with radiologic diagnosis of spondylolysis or spondylolisthesis. Spine, v. 22, n. 24, p. 2959-2967, 1997.

O'SULLIVAN, P. B. Lumbar segmental 'instability': clinical presentation and specific stabilizing exercise management. Manual Therapy, v. 5, n. 1, p. 2-12, 2000.

PACCINI, M. K.; CYRINO, E. S.; GLANER, M. F. Efeito de exercícios contra-resistência na postura de mulheres. Revista de educação física, n. 18, p. 169-175, 2007.

RICHARDSON, C. A.; JULL, G. A. Muscle control - pain control. What exercises would you prescribe? Manual Therapy, n. 1, p. 2-10, 1995.

RODRIGUES, B. G. S. et al. Pilates method in personal autonomy, static balance and quality of life of elderly females. Journal of Bodywork \& Movement Therapies. p. 1-8, 2010.

RYDEARD, R.; LEGER, A.; SMITH, D. On subjects with nonspecific chronic low back pain and functional disability: a randomized controlled trial. Journal of orthopaedic and sports physical therapy, n. 36, p. 372-484, 2006.

SACCO, I. C. N. et al. Análise biomecânica e cinesiológica de posturas mediante fotografia digital: estudo de casos. Revista brasileira de ciência \& movimento, v. 11, n. 2, p. 25-33, 2003.

SACCO, I. C. N. et al. Método pilates em revista: aspectos biomecânicos de movimentos específicos para reestruturação postural: estudos de caso. Revista brasileira de ciência \& movimento, n. 13, p. 65-78, 2005.

SEGAL, N. A.; HEIN, J.; BASFORD, J. R. The Effects of Pilates Training on Flexibility and Body Composition: An Observational Study. Archives of physical medicine and rehabilitation, v. 85, p. 1977-81, 2004.

SEKENDIZ, B. et al. Effects of Pilates exercise on trunk strength, endurance and flexibility in sedentary adult females. Journal of bodywork and movement therapies, n. 11, p. 318-326, 2007.

SILVA, A. C.; MANNRICH, G. Pilates na reabilitação: uma revisão sistemática.

Fisioterapia em Movimento, v. 22, n. 3, p. 449455, 2009.

SILVA, M. C.; FASSA, A. G.; VALLE, N. C. J. Dor lombar crônica em uma população adulta do Sul do Brasil: prevalência e fatores associados.

Cadernos de saúde pública, n. 20, p. 377-385, 2004.

THOMAS, J. R.; NELSON, J. K.; SILVERMAN, S. $\underline{\mathrm{J}}$. Métodos de pesquisa em educação física. 5 . ed. Porto Alegre: Artmed, 2007.

\section{WORLD MEDICAL ASSOCIATION. World}

Medical Association Declaration Of Helsinki: ethical principles for medical research involving human subjects. 59. ed. Seoul: WMA General Assembly, 2008.

Endereço:

Maria Erivânia Alves de Araújo

Rua Projetada, S/N, Condomínio ZEUS III, Quadra 06, Casa 07, Bairro Turú

São Luís MA Brasil

65065-720

Telefone: (98)8119.0070 (98)3081.1390

e-mail: erivania_70@hotmail.com

Recebido em: 16 de dezembro de 2009.

Aceito em: 13 de junho de 2010.

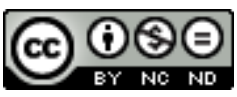

Motriz. Revista de Educação Física. UNESP, Rio Claro, SP, Brasil - elSSN: 1980-6574 - está licenciada sob Licenca Creative Commons 